\title{
THE LARGE SIEVE WITH QUADRATIC AMPLITUDE
}

STEPHAN BAIER

Abstract: We establish a large sieve bound for expressions of the form

$$
\sum_{r=1}^{R}\left|\sum_{M<n \leqslant M+N} a_{n} e\left(\alpha_{r} f(n)\right)\right|^{2},
$$

where $f(x)=\alpha x^{2}+\beta x+\theta \in \mathbb{R}[x]$ is a quadratic polynomial with $\alpha>0$ and $\beta \geqslant 0$. We also consider the case when $f(x)=x^{d}$ with $d \in \mathbb{N}, d \geqslant 3$

Keywords: large sieve, quadratic amplitude, double large sieve, exponential sums.

\section{Introduction}

Throughout this paper, we suppose that $Q, R, M, N$ are integers with $Q \geqslant 1$, $R \geqslant 1, N \geqslant 1$ and $M \geqslant 0$. As usual, by $\varepsilon$ we denote a fixed but arbitrary (small) positive real number. Further, we suppose that $\left(a_{n}\right)$ and $\left(\alpha_{r}\right)$ are sequences of complex numbers. We set

$$
S(\alpha):=\sum_{M<n \leqslant M+N} a_{n} e(\alpha n)
$$

and

$$
Z:=\int_{0}^{1}|S(\alpha)|^{2} \mathrm{~d} \alpha=\sum_{M<n \leqslant M+N}\left|a_{n}\right|^{2} .
$$

By $\|x\|$ we denote the distance of a real number $x$ to its closest integer.

In its modern form, the large sieve is an inequality connecting a discrete and the continuous mean value $Z$ of the trigometrical polynomial $S(\alpha)$, i.e. an inequality of the form

$$
\sum_{r=1}^{R}\left|S\left(\alpha_{r}\right)\right|^{2} \leqslant \Delta\left(N ; \alpha_{1}, \ldots, \alpha_{r}\right) Z
$$

2000 Mathematics Subject Classification: 11N35, 11L07. 
Montgomery and Vaughan [9] proved that (1) holds with

$$
\Delta\left(N ; \alpha_{1}, \ldots, \alpha_{r}\right)=N+\delta^{-1},
$$

where

$$
\delta:=\min _{\substack{r, s \leqslant R \\ r \neq s}}\left\|\alpha_{r}-\alpha_{s}\right\| .
$$

In many applications, the sequence $\alpha_{1}, \ldots, \alpha_{R}$ consists of Farey fractions. If $\alpha_{1}, \ldots, \alpha_{R}$ is the sequence of all fractions $a / q$ with $1 \leqslant a \leqslant q,(a, q)=1$ and $q \leqslant Q$, then the above results implies that

$$
\sum_{q \leqslant Q} \sum_{\substack{a=1 \\(a, q)=1}}^{q}\left|S\left(\frac{a}{q}\right)\right|^{2} \leqslant\left(N+Q^{2}\right) Z,
$$

which is a sharpened version of the classical large sieve inequality of Bombieri [2].

In [11] L. Zhao dealt with sums of the form

$$
\sum_{q \leqslant Q} \sum_{\substack{a=1 \\(a, q)=1}}^{q}\left|\sum_{M<n \leqslant M+N} a_{n} e\left(\frac{a f(n)}{q}\right)\right|^{2},
$$

where

$$
f(x)=\alpha x^{2}+\beta x+\theta \in \mathbb{R}[x]
$$

is a quadratic polynomial with $\alpha \neq 0$. Without loss of generality, we can assume that $\alpha>0$ (if $\alpha<0$, then we just need to replace $f(x)$ by $-f(x)$ ), which we suppose from now on.

For the case when $\beta / \alpha$ is rational, Zhao established the following bound (Theorem 2. in [11]): If $\beta / \alpha=u / v$ with $u, v \in \mathbb{Z}, v>0$ and $(u, v)=1$, then

$$
\begin{aligned}
& \sum_{q \leqslant Q} \sum_{\substack{a=1 \\
(a, q)=1}}^{q}\left|\sum_{M<n \leqslant M+N} a_{n} e\left(\frac{a f(n)}{q}\right)\right|^{2} \\
& \ll\left(Q^{2}+Q \sqrt{\alpha N(M+N+u / v)+1}\right) \Pi Z,
\end{aligned}
$$

where

$$
\Pi=\left(\frac{v}{\alpha}+1\right)^{1 / 2+\varepsilon}[N v(M+N)+|u|+v / \alpha]^{\varepsilon} .
$$

We recall that we here suppose $M \geqslant 0$.

Zhao also dealt with the case when $\beta / \alpha$ is a general real number (see Proposition 1 in [11]). However, for irrational $\beta / \alpha$ his result is weaker than (3) unless $\beta / \alpha$ is in a sense well-approximable by rational numbers. 
In many applications, the quantity

$$
Z^{*}:=N \max _{M<n \leqslant M+N}\left|a_{n}\right|^{2}
$$

does not exceed the quantity $Z=\sum_{M<n \leqslant M+N}\left|a_{n}\right|^{2}$ much. In the present paper we are concerned with large sieve inequalities of the form

$$
\sum_{r=1}^{R}\left|\sum_{M<n \leqslant M+N} a_{n} e\left(\alpha_{r} f(n)\right)\right|^{2} \ll \Delta\left(M, N ; \alpha_{1}, \ldots, \alpha_{r}\right) Z^{*} .
$$

To avoid technical complications, we confine ourselves to the case when $\beta \geqslant 0$. Though, our method should lead to the same result for $\beta<0$. We shall prove

Theorem 1. Define $\delta$ as in (2) and $Z^{*}$ as in (4). Let $f(x)=\alpha x^{2}+\beta x+\theta \in \mathbb{R}[x]$, where $\alpha>0$ and $\beta \geqslant 0$. Then we have, with an absolute $\ll-c o n s t a n t$,

$$
\begin{aligned}
& \sum_{r=1}^{R}\left|\sum_{M<n \leqslant M+N} a_{n} e\left(\alpha_{r} f(n)\right)\right|^{2} \\
& \ll\left(1+\alpha^{-1 / 2}\right) R^{1 / 2}\left(N^{1 / 2}(M+N)^{1 / 2}+\delta^{-1 / 2}\right) Z^{*} \times \log ^{1 / 2}\left(2+\alpha^{-1}\right) \log 2 N
\end{aligned}
$$

if $N>N_{0}$, where $N_{0}$ is a non-negative constant which depends only on $\alpha$ and $\beta$.

An immediate consequence of Theorem 1 is

Corollary 1. Define $Z^{*}$ as in (4). Let $f(x)=\alpha x^{2}+\beta x+\theta \in \mathbb{R}[x]$, where $\alpha>0$ and $\beta \geqslant 0$. Then we have, with an absolute $\ll-c o n s t a n t$,

$$
\begin{aligned}
& \sum_{q \leqslant Q} \sum_{\substack{a=1 \\
(a, q)=1}}^{q}\left|\sum_{M<n \leqslant M+N} a_{n} e\left(\frac{a f(n)}{q}\right)\right|^{2} \\
& \ll\left(1+\alpha^{-1 / 2}\right)\left(Q N^{1 / 2}(M+N)^{1 / 2}+Q^{2}\right) Z^{*} \times \log ^{1 / 2}\left(2+\alpha^{-1}\right) \log 2 N
\end{aligned}
$$

if $N>N_{0}$, where $N_{0}$ is a non-negative constant which depends only on $\alpha$ and $\beta$.

In the following two sections we shall prove Theorem 1. In the last section we shall touch the case of polynomials $f(x)$ of degree $\geqslant 3$.

\section{Preliminaries}

Like Zhao's method in [11], our method relies on the double large sieve of Bombieri and Iwaniec (Lemma 5.2 in [1]). Here we state only the one-dimensional version of the double large sieve. 
Proposition 1. Suppose that $x_{1}, \ldots, x_{R}$ and $y_{1}, \ldots, y_{S}$ are real numbers with

$$
-\frac{X}{2} \leqslant x_{r} \leqslant \frac{X}{2}, \quad-\frac{Y}{2} \leqslant y_{s} \leqslant \frac{Y}{2}
$$

for $r=1, \ldots, R$ and $s=1, \ldots, S$. Put $\Lambda(x):=\max (1-|x|, 0)$. Then we have

$$
\left|\sum_{r=1}^{R} \sum_{s=1}^{S} c_{r} d_{s} e\left(x_{r} y_{s}\right)\right|^{2} \leqslant\left(\frac{\pi}{2}\right)^{4} A B(X Y+1),
$$

where

$$
A:=\sum_{r=1}^{R} \sum_{\rho=1}^{R} c_{r} c_{\rho} \Lambda\left(\left(x_{r}-x_{\rho}\right) Y\right)
$$

and

$$
B:=\sum_{s=1}^{S} \sum_{\sigma=1}^{S} d_{s} d_{\sigma} \Lambda\left(\left(y_{s}-y_{\sigma}\right) X\right) .
$$

Using Proposition 1, we shall reduce the problem in question to estimating the number of solutions $k, l, u, v \in \mathbb{Z}$ of a Diophantine inequality of the form

$$
|l(v+\gamma)-k(u+\gamma)| \leqslant h,
$$

where $h$ and $\gamma$ are fixed real numbers, and the variables $k, l, u, v$ lie in certain intervals. We shall employ the following bound which is essentially due to G. Harman.

Proposition 2. Let $\gamma \in \mathbb{R}$ and $h, K, L, U, V \geqslant 1$ be given. Then the number of solutions $k, l, u, v \in \mathbb{Z}$ with $K \leqslant k \leqslant 2 K, L \leqslant l \leqslant 2 L, U \leqslant u \leqslant 2 U, V \leqslant v \leqslant 2 V$ of the inequality (8) is

$$
\begin{aligned}
& \ll\left(\min \{K, L\} \max \{U, V\}(1+|\log K / L|)+(K+L)^{3 / 2+\varepsilon}\right) \\
& \quad \times h \log 2 h \log 2(K+L),
\end{aligned}
$$

where the implied «-constant depends only on $\varepsilon$.

G. Harman stated and used the bound (9) for $U=V$ in the proof of Lemma 3 in [4] (note that our notations differ from those in [4]). He did not prove this bound in [4] but refered to his paper [3] in which he established a similar bound, Lemma 7 , for irrational real $\gamma^{\prime}$ 's which satisfy the condition

$$
\|q \gamma\|>A^{-q}, \quad \text { all } q \in \mathbb{N},
$$

for some $A$. Proposition 2 can also be established by the method used to prove Lemma 7 in [3]. Instead of the estimate (5.6) in [3] one here uses the slightly weaker estimate $\ll h T l^{-1}$ (see the remark at the beginning of the proof of Lemma 8 in [3]) which is satisfied for all real $\gamma$. We also note that the term $h^{2}$ in (5.3) in [3] can be replaced by $h \log 2 h$ (however, for the application in [3] it was sufficient to use (5.3) with $h^{2}$ ). The term $h^{2}$ arose from the crude estimate $1+\log h \ll h$ at the end of the proof of Lemma 7 in [3].

We shall also need the following slightly modified version of Proposition 2, which can be established by the same method. 
Proposition 3. Let $\gamma \in \mathbb{R}$ and $h, K, L, U, Z \geqslant 1$ be given. Suppose that $Z \leqslant U$. Then the number of solutions $k, l, u, v \in \mathbb{Z}$ with $K \leqslant k \leqslant 2 K, L \leqslant l \leqslant 2 L$, $U \leqslant u \leqslant U+Z, U \leqslant v \leqslant U+Z$ of the inequality (8) is

$$
\begin{aligned}
\ll & \left(\min \{K, L\} Z(1+|\log K / L|)+(K+L)^{3 / 2+\varepsilon}\right) \\
& h \log 2 h \log (K+L),
\end{aligned}
$$

where the implied «-constant depends only on $\varepsilon$.

\section{Proof of Theorem 1}

We are now ready to prove Theorem 1, our main result. As in [11], we begin with applying the double large sieve.

Multiplying out the square, we get

$$
\begin{aligned}
& \sum_{r=1}^{R}\left|\sum_{M<n \leqslant M+N} a_{n} e\left(\alpha_{r} f(n)\right)\right|^{2} \\
& =\sum_{r=1}^{R} \sum_{M<m \leqslant M+N} \sum_{M<n \leqslant M+N} a_{m} \overline{a_{n}} e\left(\alpha_{r}(f(m)-f(n))\right) \\
& =\sum_{r=1}^{R} \sum_{M<m \leqslant M+N} \sum_{M<n \leqslant M+N} a_{m} \overline{a_{n}} e\left(\alpha_{r} \alpha(m-n)(m+n+\beta / \alpha)\right) .
\end{aligned}
$$

In the remaining part of this paper, we assume without loss of generality that

$$
-1 / 2 \leqslant \alpha_{r} \leqslant 1 / 2
$$

for $r=1, \ldots, R$, and we put $\gamma:=\beta / \alpha$. Then, applying Proposition 1 with

$$
\begin{gathered}
\left(x_{r}\right)_{1 \leqslant r \leqslant R}=\left(\alpha \alpha_{r}\right)_{1 \leqslant r \leqslant R}, \quad\left(y_{s}\right)_{1 \leqslant s \leqslant S}=((m-n)(m+n+\gamma))_{M<m, n \leqslant M+N}, \\
\left(c_{r}\right) \equiv 1, \quad\left(d_{s}\right)_{1 \leqslant s \leqslant S}=\left(a_{m} \overline{a_{n}}\right)_{M<m, n \leqslant M+N}, \quad X=\alpha, \quad Y=2 N(M+N+\gamma),
\end{gathered}
$$

we obtain

$$
\begin{aligned}
& \left|\sum_{r=1}^{R} \sum_{M<m \leqslant M+N} \sum_{M<n \leqslant M+N} a_{m} \overline{a_{n}} e\left(\alpha_{r} \alpha(m-n)(m+n+\gamma)\right)\right|^{2} \\
& \ll A B(\alpha N(M+N+\gamma)+1) \max _{M<n \leqslant M+N}\left|a_{n}\right|^{4},
\end{aligned}
$$

where $A$ is the number of solutions $\alpha_{r}, \alpha_{\rho}$ with $1 \leqslant r, \rho \leqslant R$ of the inequality

$$
\left|\alpha_{r}-\alpha_{\rho}\right| \leqslant \frac{1}{2 \alpha N(M+N+\gamma)},
$$


and $B$ is the number of solutions $m_{1}, n_{1}, m_{2}, n_{2} \in \mathbb{Z}$ with $M<m_{1}, n_{1}, m_{2}$, $n_{2} \leqslant M+N$ of the inequality

$$
\left|\left(m_{1}-n_{1}\right)\left(m_{1}+n_{1}+\gamma\right)-\left(m_{2}-n_{2}\right)\left(m_{2}+n_{2}+\gamma\right)\right| \leqslant 1 / \alpha .
$$

Since the sequence $\alpha_{1}, \ldots, \alpha_{R}$ is well-spaced with spacing $\delta$, we have

$$
A \leqslant R\left(1+\frac{1}{\delta \alpha N(M+N+\gamma)}\right) .
$$

Obviously, $B$ is $\leqslant$ the number $B^{\prime}$ of solutions $k, l, u, v \in \mathbb{Z}$ with

$$
-2 N \leqslant k, l \leqslant 2 N, \quad 2 M<u, v \leqslant 2(M+N)
$$

of the inequality

$$
|l(v+\gamma)-k(u+\gamma)| \leqslant 1+1 / \alpha .
$$

In the following, we derive an estimate for $B^{\prime}$. We always suppose that the conditions in (15) are satisfied.

Case 1: If $k=0$, then (16) has

$$
\ll N \sum_{2 M<v \leqslant 2(M+N)}\left(1+\frac{1+\alpha^{-1}}{v+\gamma}\right) \ll N^{2}+N\left(1+\alpha^{-1}\right) \log 2 N
$$

solutions $(l, u, v)$.

Case 2: Similarly, if $l=0$, then (16) has $\ll N^{2}+N\left(1+\alpha^{-1}\right) \log 2 N$ solutions $(k, u, v)$.

Case 3: Suppose that $k<0$ and $l>0$. Then a crude bound for the number of solutions $k, l, u, v$ of (16) is

$$
\ll\left(\sum_{1 \leqslant t \leqslant 1+1 / \alpha} d(t)\right)^{2} \ll\left(1+\alpha^{-1}\right)^{2} \log ^{2} 2\left(1+\alpha^{-1}\right),
$$

where $d(t)$ is the number of divisors of $t$.

Case 4: Suppose that $k>0$ and $l<0$. Then, like in Case 3, there are $\ll\left(1+\alpha^{-1}\right)^{2} \log ^{2} 2\left(1+\alpha^{-1}\right)$ solutions $k, l, u, v$ of $(16)$.

Case 5: Suppose that $k>0, l>0$ and $M \geqslant N$. Put $J:=\left[\log _{2} N\right]+1$. Then, by Proposition 3, the number of solutions $k, l, u, v$ of (16) is

$$
\begin{aligned}
\ll & \sum_{i=0}^{J} \sum_{j=0}^{J}\left(\min \left\{\frac{N}{2^{i}}, \frac{N}{2^{j}}\right\} N\left(1+\left|\log \left(2^{j} / 2^{i}\right)\right|\right)+N^{3 / 2+\varepsilon}\right) \\
& \times\left(1+\alpha^{-1}\right) \log 2\left(1+\alpha^{-1}\right) \log 2 N \\
\ll & \left(N^{3 / 2+2 \varepsilon}+N^{2} \sum_{i=0}^{J} \sum_{j=0}^{J} \min \left\{2^{-i}, 2^{-j}\right\}(1+|j-i|)\right) \\
& \times\left(1+\alpha^{-1}\right) \log 2\left(1+\alpha^{-1}\right) \log 2 N .
\end{aligned}
$$


The double sum in the last line of (17) can be estimated by

$$
\begin{aligned}
& \sum_{i=0}^{J} \sum_{j=0}^{J} \min \left\{2^{-i}, 2^{-j}\right\}(1+|j-i|) \\
& \ll \sum_{i=0}^{J} \sum_{j=i}^{J} 2^{-j}(1+j-i) \\
& \ll \sum_{i=0}^{J} \sum_{j=i}^{J}\left(\frac{2}{3}\right)^{j} \\
& \ll \sum_{i=0}^{J}\left(\frac{2}{3}\right)^{i}
\end{aligned}
$$

and the sum in the last line of (18) is bounded by a constant. So the number of solutions in question is

$$
\ll N^{2}\left(1+\alpha^{-1}\right) \log 2\left(1+\alpha^{-1}\right) \log 2 N .
$$

Case 6: Suppose that $k>0, l>0$ and $M<N$. Put $J:=\left[\log _{2} N\right]+1$. Then, by Proposition 2, the number of solutions $k, l, u, v$ of $(16)$ is

$$
\begin{aligned}
& \ll\left(1+\alpha^{-1}\right) \log 2\left(1+\alpha^{-1}\right) \log 2 N \sum_{i=0}^{J} \sum_{j=0}^{J} \sum_{f=0}^{J+1} \sum_{g=0}^{J+1} \\
& \left(\min \left\{\frac{N}{2^{i}}, \frac{N}{2^{j}}\right\} \max \left\{\frac{M+N}{2^{f}}, \frac{M+N}{2^{g}}\right\}\left(1+\left|\log \left(2^{j} / 2^{i}\right)\right|\right)+N^{3 / 2+\varepsilon}\right) .
\end{aligned}
$$

In a similar manner like in Case 5 one proves that the expression in (19) is

$$
\ll N^{2}\left(1+\alpha^{-1}\right) \log 2\left(1+\alpha^{-1}\right) \log ^{2} 2 N .
$$

Case 7: Suppose that $k<0, l<0$ and $M \geqslant N$. Then we get the same bound like in Case 5.

Case 8: Suppose that $k<0, l<0$ and $M<N$. Then we get the same bound like in Case 6 .

Collecting all contributions together, we find that the total number of solutions $k, l, u, v$ of (16) is

$$
\ll N^{2}\left(1+\alpha^{-1}\right) \log 2\left(1+\alpha^{-1}\right) \log ^{2} 2 N
$$

if $N>N_{0}(\alpha)$, where $N_{0}(\alpha)$ is a non-negative constant which depends only on $\alpha$.

Now, combining (12), (13), (14) and the bound (20) for the term $B$, we obtain the result of Theorem 1 . 


\section{Polynomials of higher degree}

In this section we deal with the simplest polynomials of higher degree, namely the polynomials $f(x)=x^{d}$ with $d \geqslant 3$. Our aim is to estimate the expression

$$
\sum_{r=1}^{R}\left|\sum_{M<n \leqslant M+N} a_{n} e\left(\alpha_{r} n^{d}\right)\right|^{2}
$$

For simplicity, we confine ourselves to the case when $M=0$. In what follows, we allow the implied «-constants to depend on $d$ and on some parameter $k$ which we introduce below.

Using Hölder's inequality, we get for $k \geqslant 2$

$$
\sum_{r=1}^{R}\left|\sum_{n=1}^{N} a_{n} e\left(\alpha_{r} n^{d}\right)\right|^{2} \leqslant R^{1-2 / k}\left(\sum_{r=1}^{R}\left|\sum_{n=1}^{N} a_{n} e\left(\alpha_{r} n^{d}\right)\right|^{k}\right)^{2 / k} .
$$

If $k \in \mathbb{N}$, then

$$
\begin{aligned}
& \sum_{r=1}^{R}\left|\sum_{n=1}^{N} a_{n} e\left(\alpha_{r} n^{d}\right)\right|^{k} \\
& =\sum_{r=1}^{R}\left|\sum_{n_{1}=1}^{N} \ldots \sum_{n_{k}=1}^{N} a_{n_{1}} \cdots a_{n_{k}} e\left(\alpha_{r}\left(n_{1}^{d}+\ldots+n_{k}^{d}\right)\right)\right| \\
& =\sum_{r=1}^{R} \sum_{n_{1}=1}^{N} \ldots \sum_{n_{k}=1}^{N} \epsilon_{r} a_{n_{1}} \cdots a_{n_{k}} e\left(\alpha_{r}\left(n_{1}^{d}+\ldots+n_{k}^{d}\right)\right)
\end{aligned}
$$

for suitable complex $\epsilon_{r}$ with $\left|\epsilon_{r}\right|=1$.

Applying Proposition 1 with

$$
\begin{gathered}
\left(x_{r}\right)_{1 \leqslant r \leqslant R}=\left(\alpha_{r}\right)_{1 \leqslant r \leqslant R}, \quad\left(y_{s}\right)_{1 \leqslant s \leqslant S}=\left(n_{1}^{d}+\ldots+n_{k}^{d}\right)_{0<n_{1}, \ldots, n_{k} \leqslant N}, \\
\left(c_{r}\right)_{1 \leqslant r \leqslant R}=\left(\epsilon_{r}\right)_{1 \leqslant r \leqslant R}, \quad\left(d_{s}\right)_{1 \leqslant s \leqslant S}=\left(a_{n_{1}} \cdots a_{n_{k}}\right)_{0<n_{1}, \ldots, n_{k} \leqslant N}, \\
X=1, \quad Y=2 k N^{d},
\end{gathered}
$$

we obtain

$$
\begin{aligned}
& \left|\sum_{r=1}^{R} \sum_{n_{1}=1}^{N} \ldots \sum_{n_{k}=1}^{N} \epsilon_{r} a_{n_{1}} \cdots a_{n_{k}} e\left(\alpha_{r}\left(n_{1}^{d}+\ldots+n_{k}^{d}\right)\right)\right|^{2} \\
& \ll A B N^{d} \max _{n \leqslant N}\left|a_{n}\right|^{2 k},
\end{aligned}
$$

where $A$ is the number of solutions $\alpha_{r}, \alpha_{\rho}$ with $1 \leqslant r, \rho \leqslant R$ of the inequality

$$
\left|\alpha_{r}-\alpha_{\rho}\right| \leqslant \frac{1}{2 k N^{d}}
$$


and $B$ is the number of solutions $\left(m_{1}, \ldots, m_{k}, n_{1}, \ldots, n_{k}\right) \in \mathbb{N}^{2 k}$ with $m_{1}, \ldots, m_{k}$, $n_{1}, \ldots, n_{k} \leqslant N$ of the equation

$$
m_{1}^{d}+\ldots+m_{k}^{d}-\left(n_{1}^{d}+\ldots+n_{k}^{d}\right)=0 .
$$

Since the sequence $\alpha_{1}, \ldots, \alpha_{R}$ is well-spaced with spacing $\delta$, we have

$$
A \leqslant R\left(1+\frac{1}{\delta k N^{d}}\right) .
$$

Combining (21), (22), (23) and (24), we obtain

Theorem 2. Define $\delta$ as in (2). Suppose that $d, k \in \mathbb{N}, d \geqslant 3$ and $k \geqslant 2$. Then we have

$$
\sum_{r=1}^{R}\left|\sum_{n=1}^{N} a_{n} e\left(\alpha_{r} n^{d}\right)\right|^{2} \ll R^{1-1 / k}\left(N^{d / k}+\delta^{-1 / k}\right) B_{d, k}^{1 / k}(N) \max _{n \leqslant N}\left|a_{n}\right|^{2},
$$

where

$$
\begin{aligned}
B_{d, k}(N):=\mid\left\{\left(m_{1}, \ldots, m_{k}, n_{1}, \ldots, n_{k}\right) \in \mathbb{N}^{2 k}:\right. & m_{1}, \ldots, m_{k}, n_{1}, \ldots, n_{k} \leqslant N, \\
& \left.m_{1}^{d}+\ldots+m_{k}^{d}=n_{1}^{d}+\ldots+n_{k}^{d}\right\} \mid .
\end{aligned}
$$

The term $B_{d, k}(N)$ can be expressed in the form

$$
B_{d, k}(N)=\int_{0}^{1}\left|\sum_{n=1}^{N} e\left(\alpha n^{d}\right)\right|^{2 k} \mathrm{~d} \alpha,
$$

and this integral can be estimated by using Hua's inequality (see [7]). In particular, for $d=3=k$ Hua's inequality yields (see [5])

$$
B_{3,3}(N) \ll N^{7 / 2+\varepsilon} .
$$

Hooley [6] and Heath-Brown [5] established independently the much sharper bound

$$
B_{3,3}(N) \ll N^{3+\varepsilon}
$$

under the Riemann hypothesis for certain Hasse-Weil $L$-functions. Thus, Theorem 2 implies

Theorem 3. Define $\delta$ as in (2) and $Z^{*}$ as in (4). Then we have

$$
\sum_{r=1}^{R}\left|\sum_{n=1}^{N} a_{n} e\left(\alpha_{r} n^{3}\right)\right|^{2} \ll R^{2 / 3}\left(N+\delta^{-1 / 3}\right) N^{1 / 6+\varepsilon} Z^{*}
$$

If the Riemann hypothesis for Hasse-Weil L-functions holds true, then the left-hand side of (26) is

$$
\ll R^{2 / 3}\left(N+\delta^{-1 / 3}\right) N^{\varepsilon} Z^{*} .
$$


In particular, for the special case of Farey fractions we obtain

Corollary 2. Define $Z^{*}$ as in (4). Then we have

$$
\sum_{q \leqslant Q} \sum_{\substack{a=1 \\(a, q)=1}}^{q}\left|\sum_{n=1}^{N} a_{n} e\left(\frac{a n^{3}}{q}\right)\right|^{2} \ll\left(Q^{4 / 3} N+Q^{2}\right) N^{1 / 6+\varepsilon} Z^{*}
$$

If the Riemann hypothesis for Hasse-Weil L-functions holds true, then the left-hand side of $(27)$ is

$$
\ll\left(Q^{4 / 3} N+Q^{2}\right) N^{\varepsilon} Z^{*}
$$

Heuristicly, one may expect that

$$
B_{d, k}(N) \ll N^{\max \{k, 2 k-d\}+\varepsilon} .
$$

If this inequality holds, then for large $N$ the optimal choice of the parameter $k$ in Theorem 4 is $k=d$. In this case $(k=d)$ the bound (28) follows from Hooley's hypothesis $K^{*}$ in Waring's problem (see [6]) which asserts that

$$
\sum_{n \leqslant X} R_{d, d}^{2}(n) \ll X^{1+\varepsilon}
$$

where $R_{d, d}(n)$ is the number of solutions $\left(n_{1}, \ldots, n_{d}\right) \in \mathbb{N}^{d}$ of the equation

$$
n_{1}^{d}+\ldots+n_{d}^{d}=n .
$$

Thus, Theorem 2 implies

Theorem 4. Define $\delta$ as in (2) and $Z^{*}$ as in (4). Let $d \geqslant 3$ be a natural number. Assume that hypothesis $K^{*}$ holds. Then we have

$$
\sum_{r=1}^{R}\left|\sum_{n=1}^{N} a_{n} e\left(\alpha_{r} n^{d}\right)\right|^{2} \ll R^{1-1 / d}\left(N+\delta^{-1 / d}\right) N^{\varepsilon} Z^{*} .
$$

In particular, for the special case of Farey fractions we obtain

Corollary 3. Define $Z^{*}$ as in (4). Let $d \geqslant 3$ be a natural number. Assume that hypothesis $K^{*}$ holds. Then we have

$$
\sum_{q \leqslant Q} \sum_{\substack{a=1 \\(a, q)=1}}^{q}\left|\sum_{n=1}^{N} a_{n} e\left(\frac{a n^{d}}{q}\right)\right|^{2} \ll\left(Q^{2(1-1 / d)} N+Q^{2}\right) N^{\varepsilon} Z^{*}
$$

Actually, Hooley [6] and Heath-Brown [5] proved the hypothesis $K^{*}$ for $d=3$ under the Riemann hypothesis for certain Hasse-Weil $L$-functions. 
We note that for $d=2$ the bounds (29) and (30) with $\log 2 N$ in place of $N^{\varepsilon}$ follow from Theorem 1 and Corollary 1. For $d=1$ the bounds (29) and (30) with the term $N^{\varepsilon}$ omitted follow from the ordinary large sieve inequalities given at the beginning of this paper.

Acknowledgement. This paper was written when the author held a postdoctoral position at the Harish-Chandra Research Institute at Allahabad (India). The author wishes to thank this institute for financial support.

\section{References}

[1] E. Bombieri, H. Iwaniec, On the order of $\zeta(1 / 2+i t)$. Ann. Scuola Norm. Sup. Pisa Cl. Sci. (4) 13 (1986), 449-472.

[2] E. Bombieri, On the large sieve, Mathematika 12 (1965), 201-225.

[3] G. Harman, Metrical theorems on fractional parts of real sequences, J. Reine Angew. Math. 396 (1989), 192-211.

[4] G. Harman, Fractional and integral parts of $p^{\lambda}$, Acta Arith. 58 (1991), $141-152$.

[5] D.R. Heath-Brown, The circle method and diagonal cubic forms, R. Soc. Lond. Philos. Trans. Ser. A Math. Phys. Eng. Sci. 356 (1998), 673-699.

[6] C. Hooley, On Hypothesis $K^{*}$ in Waring's problem, Sieve methods, exponential sums, and their applications in number theory (Cardiff, 1995), 175-185, London Math. Soc. Lecture Note Ser. 237, Cambridge Univ. Press, Cambridge, 1997.

[7] L.K. Hua, On Waring's problem, Quart. J. Math. Oxford Ser. 9 (1938), 199-292.

[8] H.L. Montgomery, Topics in multiplicative number theory, Lecture Notes in Mathematics, Vol. 227, Springer-Verlag, Berlin-New York, 1971.

[9] H.L. Montgomery, R.C. Vaughan, The large sieve, Mathematika 20 (1973), 119-134.

[10] R.C. Vaughan, T.D. Wooley, Waring's problem: a survey, Number theory for the millennium, III (Urbana, IL, 2000), 301-340, A K Peters, Natick, MA, 2002.

[11] L. Zhao, Large Sieve Inequalities with Quadratic Amplitudes, to appear in Monatshefte für Mathematik.

Address: Queen's University, Jeffery Hall, University Ave, Kingston, ON K7L3N6 Canada

E-mail: sbaier@mast.queensu.ca

Received: 27 January 2005; revised: 13 February 2006 